\title{
End-to-End Assessment of a Large Aperture Segmented Ultraviolet Optical Infrared (UVOIR) Telescope Architecture
}

\author{
Lee Feinberg ${ }^{\mathrm{a}}$, Norman Rioux ${ }^{\mathrm{a}}$, Matthew Bolcar ${ }^{\mathrm{a}}$, Alice Liu ${ }^{\mathrm{a}}$, Olivier Guyon ${ }^{\mathrm{b}}$, \\ Chris Stark ${ }^{\mathrm{c}} ;$ Jon Arenberg ${ }^{\mathrm{d}}$

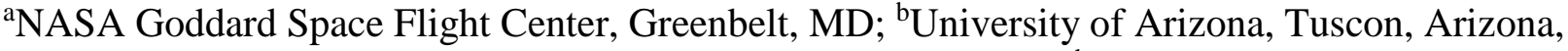 \\ USA; ${ }^{\mathrm{c} S p a c e}$ Telescope Science Institute, Baltimore, Md USA; ${ }^{\mathrm{d} N G A S, ~ R e d o n d o ~ B e a c h ~ C A ~}$
}

\begin{abstract}
Key challenges of a future large aperture, segmented Ultraviolet Optical Infrared (UVOIR) Telescope capable of performing a spectroscopic survey of hundreds of Exoplanets will be sufficient stability to achieve $10^{\wedge}-10$ contrast measurements and sufficient throughput and sensitivity for high yield Exo-Earth spectroscopic detection. Our team has collectively assessed an optimized end to end architecture including a high throughput coronagraph capable of working with a segmented telescope, a cost-effective and heritage based stable segmented telescope, a control architecture that minimizes the amount of new technologies, and an Exo-Earth yield assessment to evaluate potential performance. These efforts are combined through integrated modeling, coronagraph evaluations, and Exo-Earth yield calculations to assess the potential performance of the selected architecture. In addition, we discusses the scalability of this architecture to larger apertures and the technological tall poles to enabling it.
\end{abstract}

Keywords: ATLAST, LUVOIR, Exoplanet, HDST, Space Telescope

\section{INTRODUCTION}

One possible successor to the James Webb Space Telescope and WFIRST is an observatory that combines general Ultraviolet-Optical Infrared (UVOIR) astrophysics with the search for life on habitable Earth-like exoplanets using a large aperture segmented telescope. Work on this problem began in 2009 as a potential Advanced Telescope Large Aperture Space Telescope (ATLAST) architecture ${ }^{i}$ ii. Early work focused on a scalable $9.2 \mathrm{~m}$ segmented telescope that could launch in a Delta IV heavy and the work has recently progressed to a $12 \mathrm{~m}$ segmented telescope $\mathrm{e}^{\mathrm{iii}}$. The most significant architectural driver beyond the aperture size is the $10^{\wedge}-10$ contrast required to block out the bright stars sufficiently to detect dim earth like planets. Achieving this requires a combination of a high throughput coronagraph with sufficient bandpass and wavelength range to perform spectroscopic surveys and a very stable telescope that maintain the $<10$ picometers required for most observations. Meanwhile, the general class UVOIR science requires a large field of view telescope with sufficient diffraction limited performance at 500nm's with throughput into the ultraviolet. In the past, the UV aspects of this architecture were known to be a challenge but more recently the team has recognized that the diffraction limited wavelength performance itself is also a challenge that requires careful attention.

In the past, the efforts of the coronagraph teams and the segmented telescope teams have not been well integrated. The segmented telescope teams were able to build upon the experience and heritage of JWST and HST while the coronagraph teams were able to build upon the WFIRST and ground coronagraph heritage to offer technological solutions independent of each other. Here we try to begin the process of merging the efforts into a single end to end architecture that is optimized both for general astrophysics and Exoplanet detection. While still notional, just the existence of a single end to end architecture gives a roadmap of potential technology needs and priorities and highlights areas that will be most challenging. For the purposes of this paper, we will discuss the various aperture sizes that have been studied for packaging purposes, optical designs and coronagraph options studied for the $12 \mathrm{~m}$ High Definition Space Telescope (HDST), and stability modeling that has been done on a $9.2 \mathrm{~m}$ scalable segmented architecture. The end to end architecture provided is intended to be size invariant and thus compatible with many different aperture sizes ranging from 9.2 meters to even 20 meters but with an end to end focus on the $12 \mathrm{~m}$ class system. In the end, the final aperture size will be driven by the programmatic and scientific trades of these various options. 
The beginning point for understanding this architecture are the telescope requirements which are updated here to be consistent with the architecture presented. For example, room temperature has been chosen for the mirror to be compatible with the stability assessment provided.

\begin{tabular}{|c|c|c|c|c|}
\hline \multicolumn{2}{|l|}{ Parameter } & Requirement & Stretch Goal ${ }^{\dagger}$ & Traceability \\
\hline \multicolumn{2}{|c|}{ Primary Mirror Aperture } & $\geq 8.0$ meters & $>12+$ meters & $\begin{array}{l}\text { Resolution, } \\
\text { Sensitivity, Exoplanet } \\
\text { Yield }\end{array}$ \\
\hline \multicolumn{2}{|c|}{ Telescope Temperature } & $293 \mathrm{~K}$ & - & $\begin{array}{l}\text { Thermal Stability, } \\
\text { Integration \& Test, } \\
\text { Contamination, } \\
\text { IR Sensitivity }\end{array}$ \\
\hline \multirow{4}{*}{$\begin{array}{l}\text { Wavelength } \\
\text { Coverage }\end{array}$} & UV & $100 \mathrm{~nm}-300 \mathrm{~nm}$ & $90 \mathrm{~nm}-300 \mathrm{~nm}$ & - \\
\hline & Visible & $300 \mathrm{~nm}-950 \mathrm{~nm}$ & - & - \\
\hline & NIR & $950 \mathrm{~nm}-1.8 \mu \mathrm{m}$ & $950 \mathrm{~nm}-2.5 \mu \mathrm{m}$ & - \\
\hline & MIR & Sensitivity to $8.0 \mu \mathrm{m}^{\dagger \dagger}$ & - & Transit Spectroscopy \\
\hline \multirow{2}{*}{$\begin{array}{l}\text { Image } \\
\text { Quality }\end{array}$} & UV & $<0.20 \operatorname{arcsec}$ at $150 \mathrm{~nm}$ & - & - \\
\hline & Vis/NIR/MIR & $\begin{array}{l}\text { Diffraction-limited at } 500 \\
\mathrm{~nm}\end{array}$ & - & - \\
\hline \multicolumn{2}{|l|}{ Stray Light } & $\begin{array}{l}\text { Zodi-limited between } \\
400 \mathrm{~nm}-1.8 \mu \mathrm{m}\end{array}$ & $\begin{array}{l}\text { Zodi-limited between } \\
200 \mathrm{~nm}-2.5 \mu \mathrm{m}\end{array}$ & $\begin{array}{l}\text { Exoplanet Imaging \& } \\
\text { Spectroscopy SNR }\end{array}$ \\
\hline \multicolumn{2}{|c|}{ Wavefront Error Stability } & $\begin{array}{l}\sim 10 \text { pm RMS uncorrected } \\
\text { system WFE per } \\
\text { wavefront control step }\end{array}$ & - & $\begin{array}{l}\text { Starlight Suppression } \\
\text { via Internal } \\
\text { Coronagraph }\end{array}$ \\
\hline \multirow{2}{*}{ Pointing } & Spacecraft & $\leq 1$ milli-arcsec & - & - \\
\hline & Coronagraph & $<0.4$ milli-arcsec & - & - \\
\hline
\end{tabular}

Table 1: Large Ultraviolet Optical Infrared observatory requirements and goals

Similar to past versions of ATLAST optical design, the basic approach chosen here is a Cassegrain telescope front end coated with AlMgF sensitive down to 100nm's that feeds both a coronagraph and an Ultra-Violet instrument which we will call the Cassegrain Instruments (CI). Similar to JWST, the off-axis field is sent to a high reflectivity silver or gold coated Tertiary mirror and then a fine steering mirror which allows a large field of view over visible wavelengths and near-infrared wavelengths, these instruments are referred to as TMA instruments (TI). For the purposes of this paper, we do not elaborate on the detailed makeup of the general class science instruments which we expect to come out of the Large Ultra-Violet Optical InfraRed (LUVOIR) Science and Technology Definition Team (STDT). However, the TI design is compatible with large field of view imagers and spectrographs that operate above $400 \mathrm{~nm}$ and the CI architecture is compatible with modest field of view ultra-violet instruments and coronagraphs.

Considering the key challenge of high contrast earth like planet coronography, a key facet of the overall architecture will be the need to survey and perform spectroscopy of a large number of stars. The key metric of success in performing this survey is the Exo-earth yield calculation and details of this type of calculator can be found in papers by Stark ${ }^{\text {iv }}$. By studying the sensitivity of the yield calculator parameters, we can determine key observatory drivers that will guide the architecture design. An updated yield calculation consistent with the end to end architecture is shown below in Figure 1. A key driver on yield is the need to survey a large number of stars and to be able to make multiple visits per star. Thus, the architecture needs to have a large field of regard and needs to be stable in an efficient way to make this feasible. This will form the guiding principal of the thermal and dynamic approach taken. The throughput of the system is also critical which includes both the throughput of the segmented coronagraph over sufficient bandpasses and the telescope size and 
system throughput. A larger telescope with the highest possible throughput will be ideal for this problem and this forms a key driver in the telescope architecture presented.
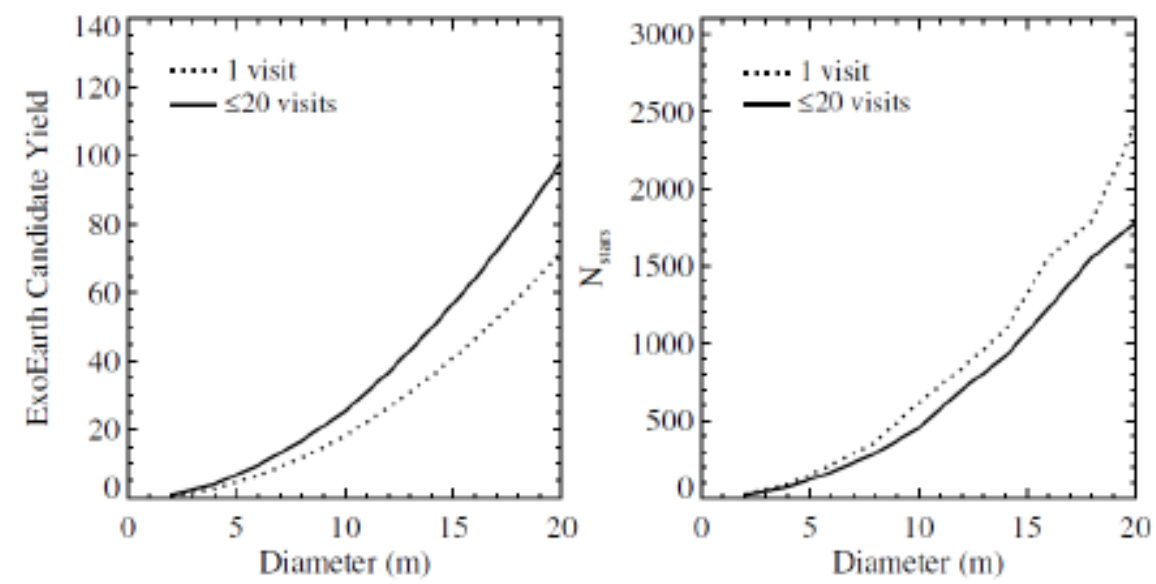

Figure 1: Yield as function of diameter (left) and number of visits required as a function of diameter (right)

The other practical consideration is how to package and deploy and ultimately build the segmented space telescope. For this, we start with the heritage of JWST which demonstrates the basic approach of both a segmented space telescope and a deployed space telescope. JWST provides a great starting point for the design in terms of both actual hardware and design heritage. The design then evolves to optimize it for the considerations provided in the previous paragraph.

\section{TELESCOPE ARCHITECTURE}

The basic telescope architecture is closely connected to the aperture size. As seen in Figure 2, we have studied several different size options for packaging and rocket options ranging from $9.2 \mathrm{~m}$ and $12 \mathrm{~m}$ in a Delta IV Heavy, Falcon 9 Heavy or SLS, $12 \mathrm{~m}$ and $15 \mathrm{~m}$ architectures in a SLS, and even 20-meter class assembled options ${ }^{\mathrm{v}}$. Closely connected to the packaging issues are the available mass and this has been studied in terms of mass carrying capability of various rockets to L2. A key point at this time is that only the Delta IV Heavy has currently flown. While a 12-meter with an aggressive packaging can possibly fit in this size shroud, the mass carrying capabilities may be a challenge. We do anticipate that the SLS Block 1 will provide significantly more mass for this option though the packaging concept will remain challenging. The SLS Block 1B with its much larger shroud (8.4 meters) would greatly simplify the packaging challenge of a $12 \mathrm{~m}$ class telescope while providing significant mass margin. However, a new fairing is a more significant development that is farther off in the future and will require other programmatic forces to support its development. 


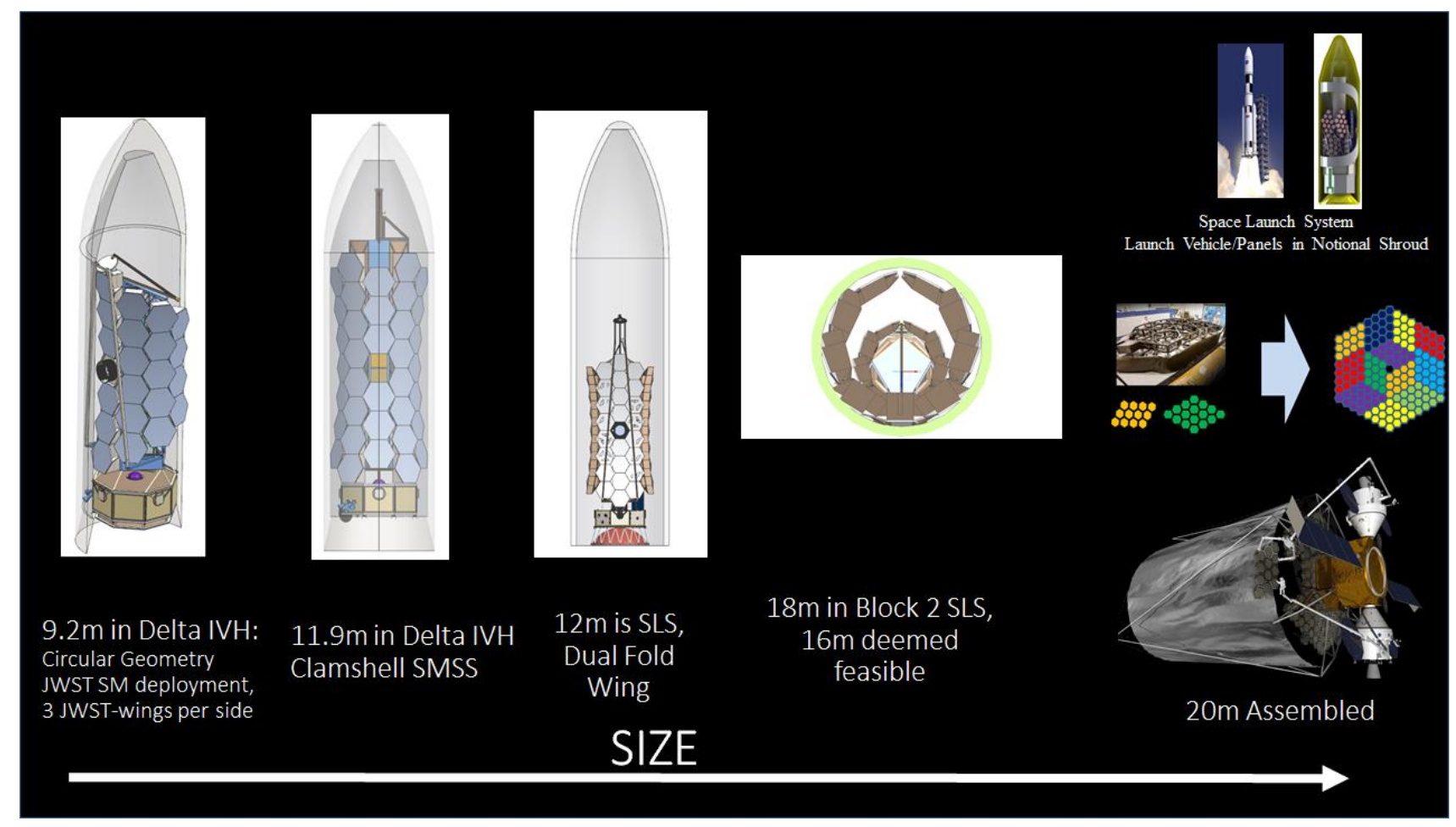

Figure 2: Packaging Options
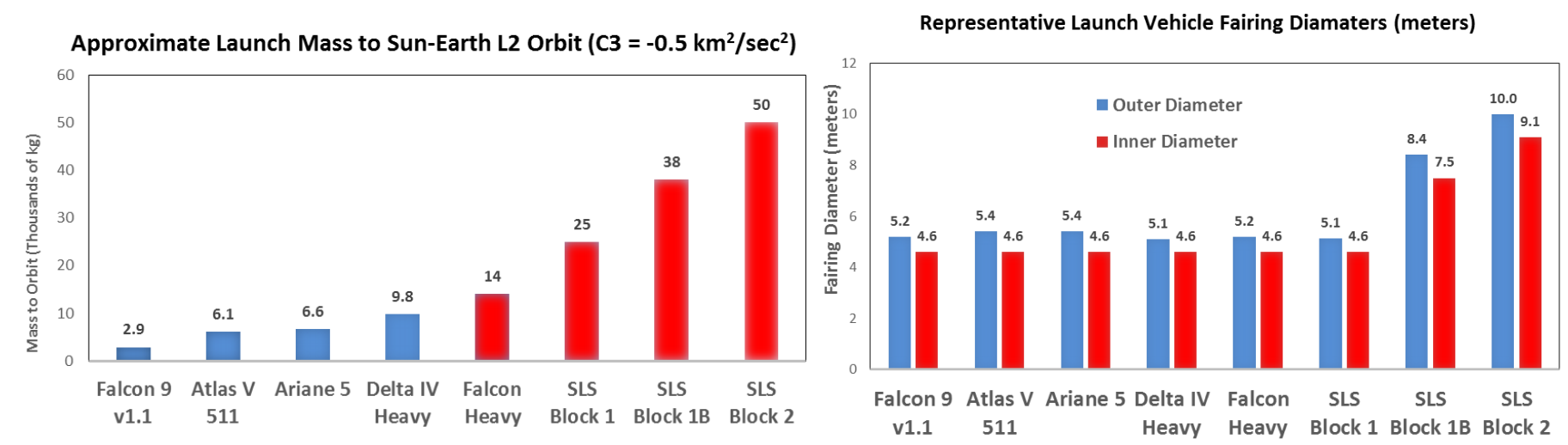

Figure 3: Mass vs. Rocket

\section{STABILITY ARCHITECTURE}

In order to accommodate any of the possible aperture sizes ranging from 9.2 meter up to 20 meter, we have developed a size invariant stability architecture that leverages JWST heritage and optimizes stability to achieve the picometer class 
performance required shown below in Figure 4. Using JWST provided a good starting point for dynamic and mechanical models which retained the potential to save costs through heritage and also provided a departure point to optimize for better performance. The team also started with the least ambitious size of 9.2 meters both to assure there is at least a solution that can use an existing rocket and because it provides an initial proof of principle at the lowest risk. If a stability solution did not exist for the 9.2 meter aperture, the team would have concluded that larger was not feasible. For these reasons the reference architecture itself is based on the JWST segment size with an additional ring of mirrors to achieve 9.2 meters but mirror temperature was chosen to be room temperature again to make the system cost-effective and based on existing mirror technologies. While several different mirror technologies have been studied including glass and Silicon carbide, in this study we use ULE based on lightweight mirror technology that was initially developed for JWST and lost out to beryllium mainly due to operating temperature considerations ${ }^{\mathrm{vi}}$. This mirror technology is also quite lightweight, has a long heritage of use for UV systems with excellent surface roughness and mid frequency, a well understood database of CTE variability, and provides a great potential starting point. Other architectures do exist that may allow an active primary mirror or which use heavier mirrors but are not the focus of this paper. Moreover, our contention is that stiff, lightweight ULE mirrors at room temperature provide the most stable segmented architecture that balances both mirror stiffness and thermal stability and that stability is the driving requirement to assure the critical $10^{\wedge} 10$ contrast is met.

$\mathrm{Fi}$

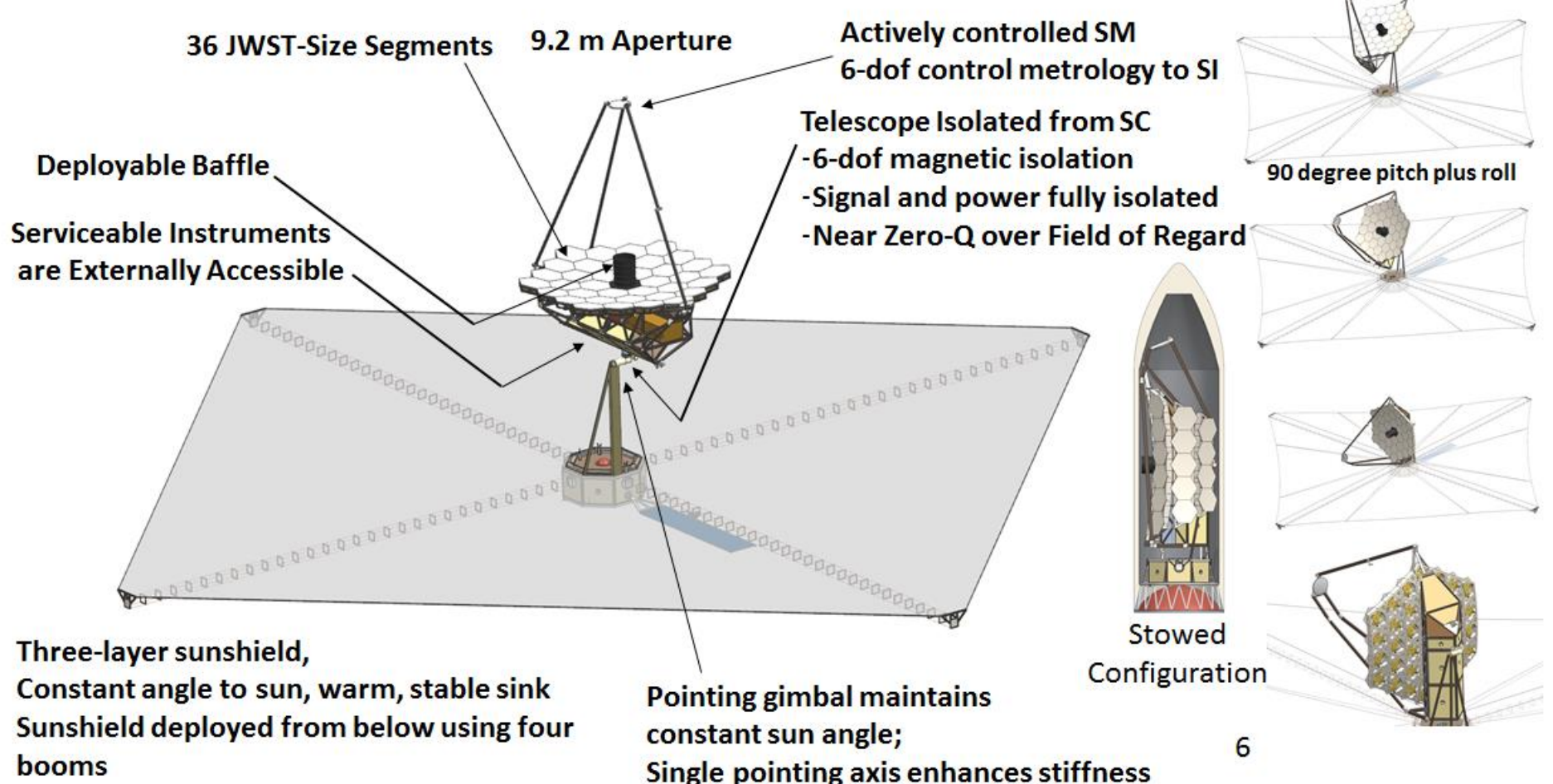

Figure 4: Overall Architecture

The architecture chosen is also an open type architecture that uses a sunshield to block sun, earth and moon similar to that used for JWST. While a deployable light baffle was considered, early stray light modeling found the baffle has a very minor impact because the limiting stray light was in-field and not from paths the baffle would block. The baffle itself also would have been a source of instability unless it is maintained at a very stable temperature which would have 
required heaters embedded in the baffle. While not fully ruled out, our determination was a planar tensioned sunshield would be the lowest cost, mass and power option and also leverages the JWST heritage.

A key aspect of the thermal architecture is to keep both the primary mirror segments and secondary mirror thermally and dynamically stable. The primary mirror segments are heated from the back and this will be discussed later. To maintain good dynamic stability over a large field of regard, we adopted to use a stiff gimbal to point the telescope and a noncontact isolation system with additional reaction wheel isolation, this approaches lets us use the full wheel speeds of the reaction wheels. The thermal stability is achieved by combining milli-Kelvin class heaters behind the mirrors and in the backplane with an architecture which is nearly zero Q as the telescope gimbal is slewed. This is because the sunshield is run cold enough $(160 \mathrm{~K})$ where the change in $\mathrm{Q}$ due to view factor changes are minimized while just above where water will deposit. For view factor changes from the secondary, our calculations showed only microwatts heat changing in the front surface of the mirrors using AlMgF coatings and our ULE architecture. For this type of actively controlled thermal architecture, stability is dominated by CTE and thermal inertia. ULE at room temperature has the lowest CTE of any mirror material at any temperature making it the ideal solution and making this architecture the most thermally stable architecture possible (thermal mass between mirror materials does not vary), thermal conductance does not play a significant role in the stability.

The mirrors themselves can be made both dynamically and thermally stable by making them stiff ( $>500 \mathrm{hz}$ goal) and through an adiabatic thermal architecture. As shown in Figure 5, there are actually several ways to combine different layers of stability controls to achieve all aspects of stability. The segment thermal and dynamic stability are controlled at that level and do not require active controls. However, segment to segment motions get more difficult to control as the diameter gets larger. Moreover, calculations show that there is not adequate signal to noise from the science star to close the control loop faster than a time scale of at least minutes using the star itself. Moreover, dimmer stars have less signal and the control loop can be as slow as hours. To address this, we have adopted a scale invariant backplane architecture that uses edge sensing coupled to a segmented deformable mirror as a notional approach to remove backplane instabilities. This approach could be used only to remove slow drifts (thermally induced) and a feed-forward type controls could be used to remove dynamic effects like jitter.

\begin{tabular}{|c|c|c|c|}
\hline & $\begin{array}{l}\text { Layer 1: Minimum } \\
\text { observatory (active heater, } \\
\text { non-contact isolation) }\end{array}$ & $\begin{array}{l}\text { Layer 2: Use internal } \\
\text { coronagraph sensing and } \\
\text { control methods }\end{array}$ & $\begin{array}{l}\text { Layer 3: Use telescope } \\
\text { metrology systems }\end{array}$ \\
\hline Segment Thermal Stability & $\begin{array}{l}\text { Low } Q \text { architecture, Active PM } \\
\text { heater control, material choice }\end{array}$ & $\begin{array}{l}\text { Zernike Sensor with continuous DM } \\
\text { control }\end{array}$ & \\
\hline $\begin{array}{l}\text { Segment to Segment Thermal } \\
\text { Stability }\end{array}$ & $\begin{array}{l}\text { Active heater and MLI control, } \\
\text { material choice, joint design }\end{array}$ & $\begin{array}{l}\text { Zernike Sensor with Continuous or } \\
\text { Segmented DM control (piston, } \\
\text { tip/tilt), Use bright star (reduce } 10 \\
\text { minute update rates) }\end{array}$ & Laser metrology, edge sensors \\
\hline Segment Dynamics Stability & $\begin{array}{l}\text { Stiffness and Design, Possibly } \\
\text { smaller segments, materials }\end{array}$ & & \\
\hline $\begin{array}{l}\text { Segment to Segment Dynamic } \\
\text { Stability }\end{array}$ & $\begin{array}{l}\text { Reaction Wheel isolators, Non- } \\
\text { contact Isolation between SC and } \\
\text { telescope, } \\
\text { Design, TMD's (if needed), material } \\
\text { choice }\end{array}$ & $\begin{array}{l}\text { Zernike Sensor, Feed forward DM } \\
\text { control, Use bright star (reduce } \\
\text { update rate) }\end{array}$ & Laser metrology, edge sensors \\
\hline Line of Sight/SM Thermal Stability & Low $Q$ architecture, Heater & $\begin{array}{l}\text { LOS sensor and control mirror, MIMF } \\
\text { for SM alignment }\end{array}$ & Laser truss, image based techniques \\
\hline Line of Sight/SM Dynamic Stability & $\begin{array}{l}\text { Reaction wheel isolators, Non- } \\
\text { contact isolation, Design, TMD (if } \\
\text { needed) }\end{array}$ & $\begin{array}{l}\text { LOS sensor and control with feed } \\
\text { forward control }\end{array}$ & $\begin{array}{l}\text { Laser truss, imaged based } \\
\text { techniques }\end{array}$ \\
\hline
\end{tabular}

Figure 5: Layered Archtiecture 
A notional active control architecture is shown in Figure 6. The key to this architecture is to only control the sensitive degrees of freedom of the primary mirror (piston, tip, tilt). Other degrees of freedom like decenter and rotation are more than 100x less sensitive and can be controlled in design. Moreover, edge sensor technologies have been maturing both for segmented apertures and in commercial applications including now the capability to measure $<10 \mathrm{pm} \mathrm{rms} \mathrm{in} \mathrm{a} 64$ channel fiber fed commercial device vii. The segmented deformable mirror itself requires picometer class stability and resolution which has been demonstrated on continuous facesheet type deformable mirrors so is believed feasible but more work will be needed on size, multiplexing, and speed.

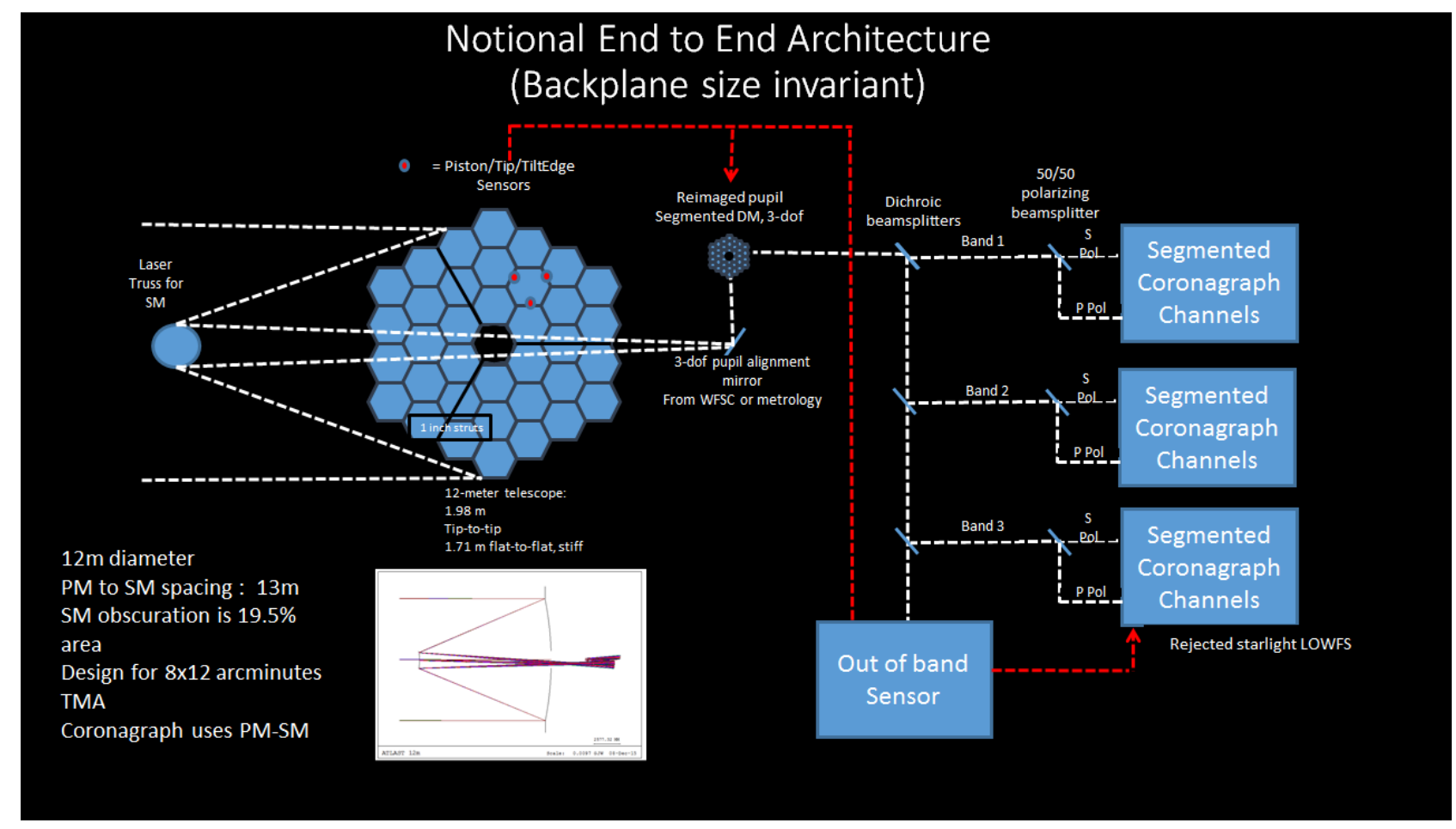

Figure 6: Active Loop Architecture

Several issues still need to be studied for this architecture including alignment sensitivities which may require additional active control, the effectiveness of dichroics, and the degree to which the use of a polarizing beamsplitter can mitigate polarization leakage. Polarization leakage can also be partially controlled through design and this will be a key future area to study.

\section{CORONAGRAPH CONSIDERATIONS}

A key aspect of this architecture is that there is a segmented coronagraph with sufficient throughput and contrast that is compatible with the architecture. This is an area that is quickly evolving and where designs are beginning to evolve that can meet the contrast and bandpass requirements. ${ }^{\text {viii }}$ Through the work of the Segmented Coronagraph Design team optimizations will be performed that will improve the throughput. In considering the observatory architectures, the team considered ways the telescope itself could be modified to improve performance. While an off-axis design is possible, the distance to the secondary mirror would greatly increase which would be quite complicated for a telescope this large and this would complicate the need to be wide field of view for general astrophysics. One telescope architecture change that does help throughput and appears feasible is to make narrower secondary mirror support struts. Our team did assess a 1 " wide but deeper strut and found it to be technically feasible and thus is now included in the integrated 
model results provided in section 7. Smaller gaps were also considered although initial feedback was that this would not make as big a difference and it is technically challenging to accomplish. Recent indications are that having a circular aperture may be useful. If so, we believe that partial mirrors could be added to circularize the geometry if needed. Segment size is also under study and a consideration. However, segment stiffness and thermal stability goes with $1 / \mathrm{D}^{2}$ so we are reluctant to go to larger segments unless it is a very significant improvement factor.

As was seen in the notional architecture, the coronagraph itself could include both polarization and dichroic beamsplitters to multiplex wavelengths. There may be clever ways to limit the number of detectors by sharing focal surfaces but clearly this type of multiplexing will mean that coronagraph channel elements like deformable mirrors and Lyot Stops will want to make use of economies of scale by employing ASIC electronics, hybridization, and mass produced DM's (like MEMS or PMN actuators). Fortunately, economies of scale will benefit this architecture as there will be one non-recurring design but recurring cost savings in the components.

The architecture shown also includes an initial segmented DM that is used to correct tip, tilt, piston. Segmented deformable mirrors are routinely made using MEMS technology. However, one could even imagine a number of small hexagonal mirrors made of stable ULE that are driven in the three degrees of freedom using the same type of stable actuators used on a continuous deformable mirrors. The key question is how fast can any one of these segmented DM technologies close the loop with picometer resolution. A key aspect of this will be the electronics. If the loop can be closed at hundreds of Hertz, one can remove segment jitter and segment drifts (thermal and jitter drift) using edge sensors. This approach will work providing the mirror is extremely stable.

Another critical consideration for the coronagraph is throughput. A quick summary of the approximate throughput of key contributors (at 500nm) is shown in the Table below. This is just an initial assessment of reflectivities and throughputs but provide reasonable approximations for yield assumptions.

\begin{tabular}{|c|c|}
\hline & Throughpu \\
\hline Primary & 0.92 \\
\hline Secondary & 0.92 \\
\hline Areal obsuration & 0.805 \\
\hline Additional gaps and struts & 0.97 \\
\hline Coronagraph Surface Reflectivity & 0.98 \\
\hline Coronagraph number of reflections & 15 \\
\hline Corongraph mirror throughput (15 surfaces) & 0.738569 \\
\hline Coronagraph Lyot/pupil thoughput & 0.4 \\
\hline TOTAL THROUGHPUT & 0.195252 \\
\hline
\end{tabular}

Table 2: Coronagraph Throughput Estimate 


\section{INTEGRATED MODELING}

A key to demonstrating the feasibility of this approach is the modeling that has been performed to substantiate the approach. Our team has been involved in 2 major modeling efforts aimed at demonstrating that a large stable telescope can be made. The first was to perform thermal stability modeling of a realizable ULE mirror and the second was to perform end to end integrated modeling of the dynamics.

In 2015, Eisnehower ${ }^{\text {ix }}$ demonstrated that with $1 \mathrm{mK}$ heater control, one can achieve roughly 1 picometer RMS stability (predicted, no model uncertainty factor) on a 1.2m ULE segment. To do this, he used actual CTE measurements of coupons taken from 2 different actual mirror boules. Depending on the model uncertainty factor assumed, this result either provides some margin or just meets our needs. Some hidden margin exists in the simple heater approach used but there is also a lot more details that are needed in how the mirror is mounted (and mount temperature control) and in edge effects and thermal implementations. These results are encouraging but just a first step and emphasize the criticality of having the maximum amount of margin possible. The CTE used came from the mirror shown in Figure 7 and the results shown in Figure 8 are for two different realizations of how to configure the mirror. Mirror 2 is a realizable approach but Mirror 1 demonstrate that even small variations in CTE can greatly impact the performance.
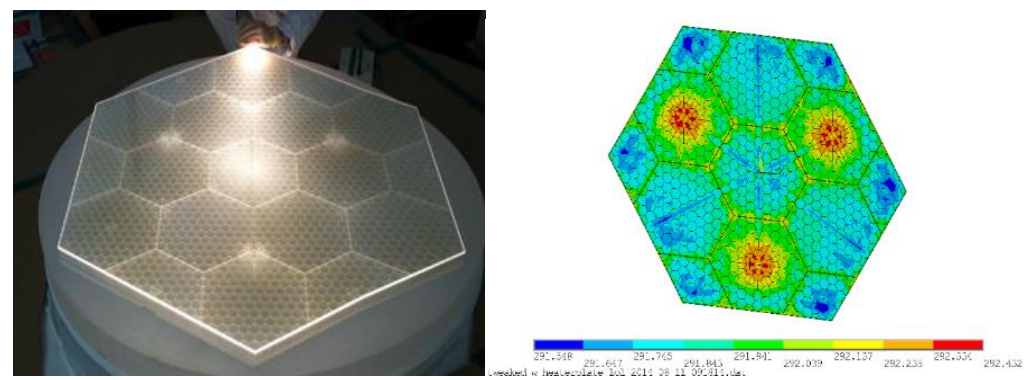

Figure 7: ULE Mirror and Associated Thermo-Mechanical-Optical Model

\begin{tabular}{|l|l|l|l|l|}
\cline { 2 - 5 } \multicolumn{1}{c|}{} & \multicolumn{2}{l|}{$98 \mathrm{mK}$ control } & \multicolumn{2}{l|}{$1.27 \mathrm{mK}$ control } \\
\hline CTE Distribution & RMS $(\mathrm{pm})$ & $\mathrm{PV}(\mathrm{pm})$ & $\mathrm{RMS}(\mathrm{pm})$ & $\mathrm{PV}(\mathrm{pm})$ \\
\hline Mirror 1 & 3.8 & 13.9 & 4.94 & 18.1 \\
\hline Mirror 2 & 0.514 & 1.82 & 0.67 & 2.38 \\
\hline
\end{tabular}

Figure 8: ULE Thermal Stability Modeling Results 
In addition to thermal stability modeling of the segments, our team has been studying the dynamics of a full scale 9.2 meter scalable telescope. This effort initially started by matching our finite element models to JWST dynamics using JWST reaction wheel disturbances as the primary steady state consideration. As the actual geometry matured, we updated the dynamics model. After several iterations, we now have a two layer isolation approach that combines a non-contact isolation which essentially floats the telescope with a passive isolation at the reaction wheels. The assumed performance of the isolation has been published and demonstrated and is believed relatively mature. Shown in Figure 9 below, this approach gives excellent stability for both the primary mirror (less than 1 picometer) and line of sight (under 1 microarcsecond). However, it should be noted that these results do not include model uncertainty factors, potential shorts and other factors like cable connections, slosh, and pointing effects. Line of sight results are so excellent that we believe it is feasible no active controls are needed for this purpose. However, primary mirror stability is a critical factor and validating and verifying these level of disturbances may prove difficult so a high speed jitter control may prove necessary.
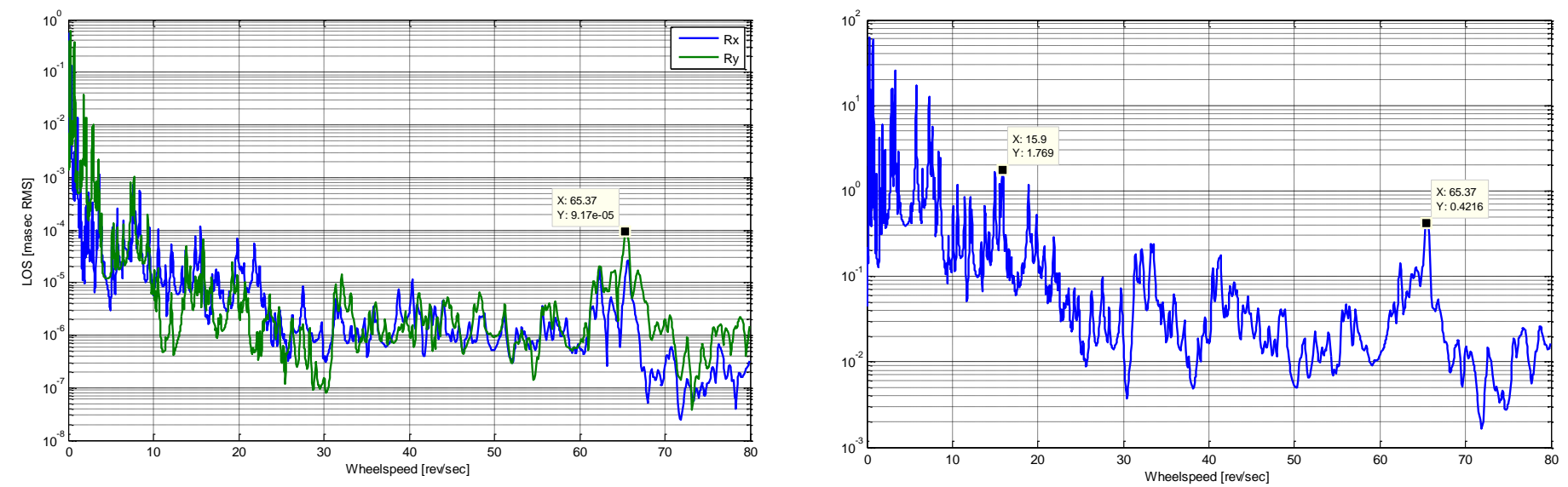

Figure 9: Line of Sight (left), WFE in picometers RMS (right)

In addition to jitter and line of sight, one also needs to consider segment deformations themselves, especially if one wants to consider high speed segment control. Our team has been studying segment dynamics on JWST mirror segments and have seen how segment motions can couple to other aberrations which we believe are the result of segment deformations. Shown below in Figure 10 , we have measured changes in mirror figure that are coupled to tip/tilt of the mirror. This work is still ongoing but Saif and team have shown coupling between mirror tip/tilt and astigmatism and more recent results are confirming the mirror itself is bending due to its own intertia when it vibrates. ${ }^{\mathrm{x}}$ Making stiffer mirrors will greatly mitigate this concern and will also assure our mirrors are well above the highest speed of the reaction wheels. Our assessment is that a 500hz first mode is a good goal and would assure the mirror would be stiff enough both for dynamic reasons but also to assure that gravity effects can be backed out and that the mirror can be polished to the rather tight tolernaces needed anyway just to be a diffraction limited telescope at 500nm's. 
Spatial Modes of a mirror segment demonstrates rigid body and deformation modes

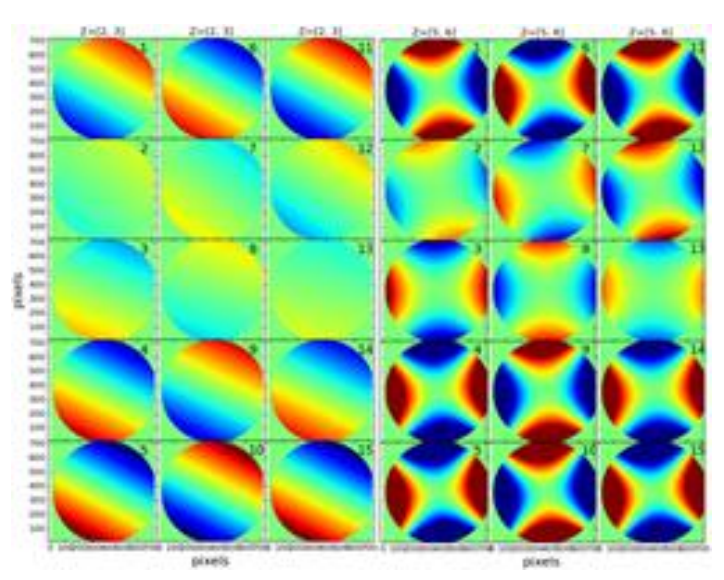

Figure 10: Demonstration of Mirror Tilting Coupling to Deformations

\section{EXTERNAL GUIDE STAR}

A new idea that has emerged is to use an external guide star in front of the telescope. The advantage of this idea is it would allow a much brighter source so that the sensing and control loop could be closed either fast enough to remove drift (eg, .1 hz) or fast enough to remove jitter (400hz). The key issue that needs to be studied is how far away would the source need to be and the associated repointing times. If close enough, it may be a rather cost effective method to move the source would be possible. More work on this is needed but the results could be paradigm shifting. If the sense and control loop could be closed fast enough, the stability requirement on the telescope could even be relaxed to the point of being just a diffraction limited telescope at 500nm's.

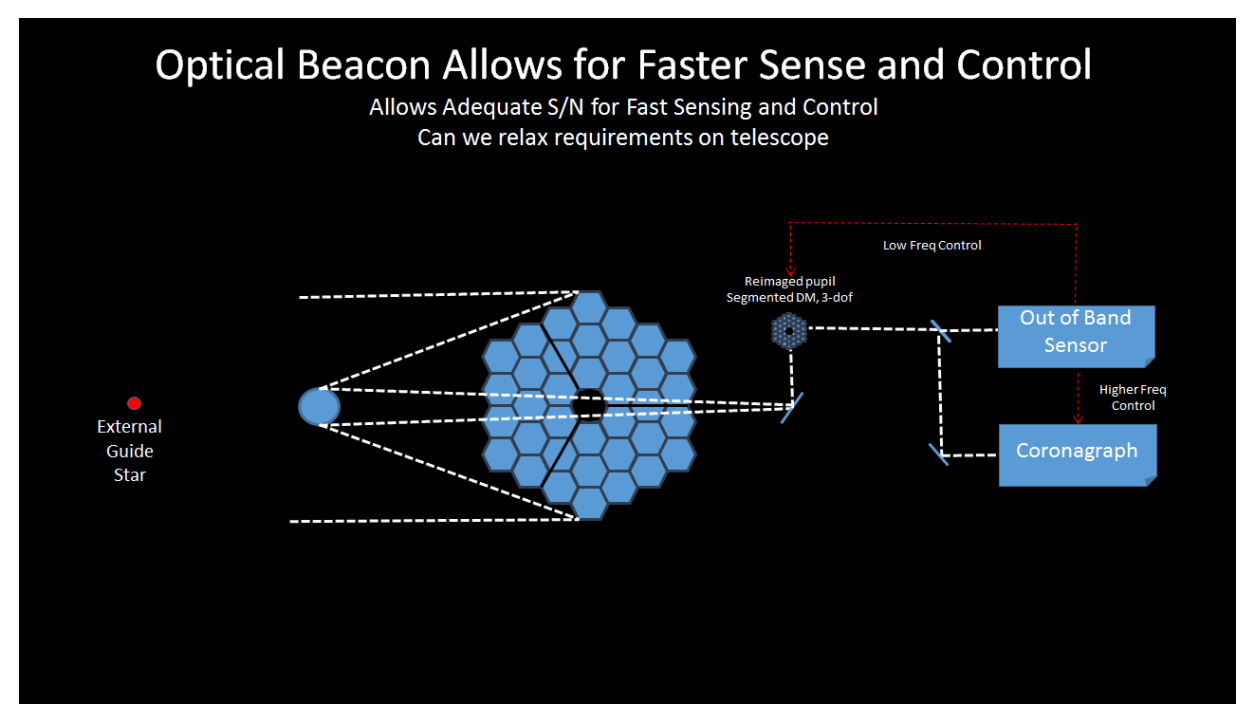

Figure 11: External Guide Star 


\section{HERITAGE}

While the observatory proposed has evolved to be optimized for both general astrophysics and Exoplanet high contrast with high yield, it still builds considerably on the heritage of JWST which will help to reduce risk and cost. Shown in Figure 11, there are many places where heritage is directly relevant. Starting in the upper right, the telescope structure design, assembly, integration and deployments can all serve as great departure points. In some cases like latches and motors and motor electronics, designs may be directly useable. The methods for integrating and aligning the primary mirror can also be directly leveraged which is the result of 10 years of past experience. Finally, the test program itself can be greatly leveraged. A 12 meter telescope can fit in the JSC vacuum chamber Cup Up though the secondary mirror would have to be deployed inside the chamber. This configuration can be used for thermal balance testing. If the telescope is room temperature, the alignments and system wavefront error can either be tested in a cleanroom or in a vacuum chamber. The methods used on JWST for testing mirrors and matching radius of curvatures would be a great departure point to get mirrors almost to their final performance requirements. The test configuration at MSFC could be used to perform mirror stability testing and even subscale segment to segment testing. Even the Pathfinder program would be a great model for a future observatory. These are just a few examples of how heritage would directly translate. There are many details like actuators, electronic designs, mirror containers, composite methods, contamination handling, dynamics modeling, integrated modeling, L2 environment and orbital analysis which all would provide great departure points and greatly reduce risk and cost.

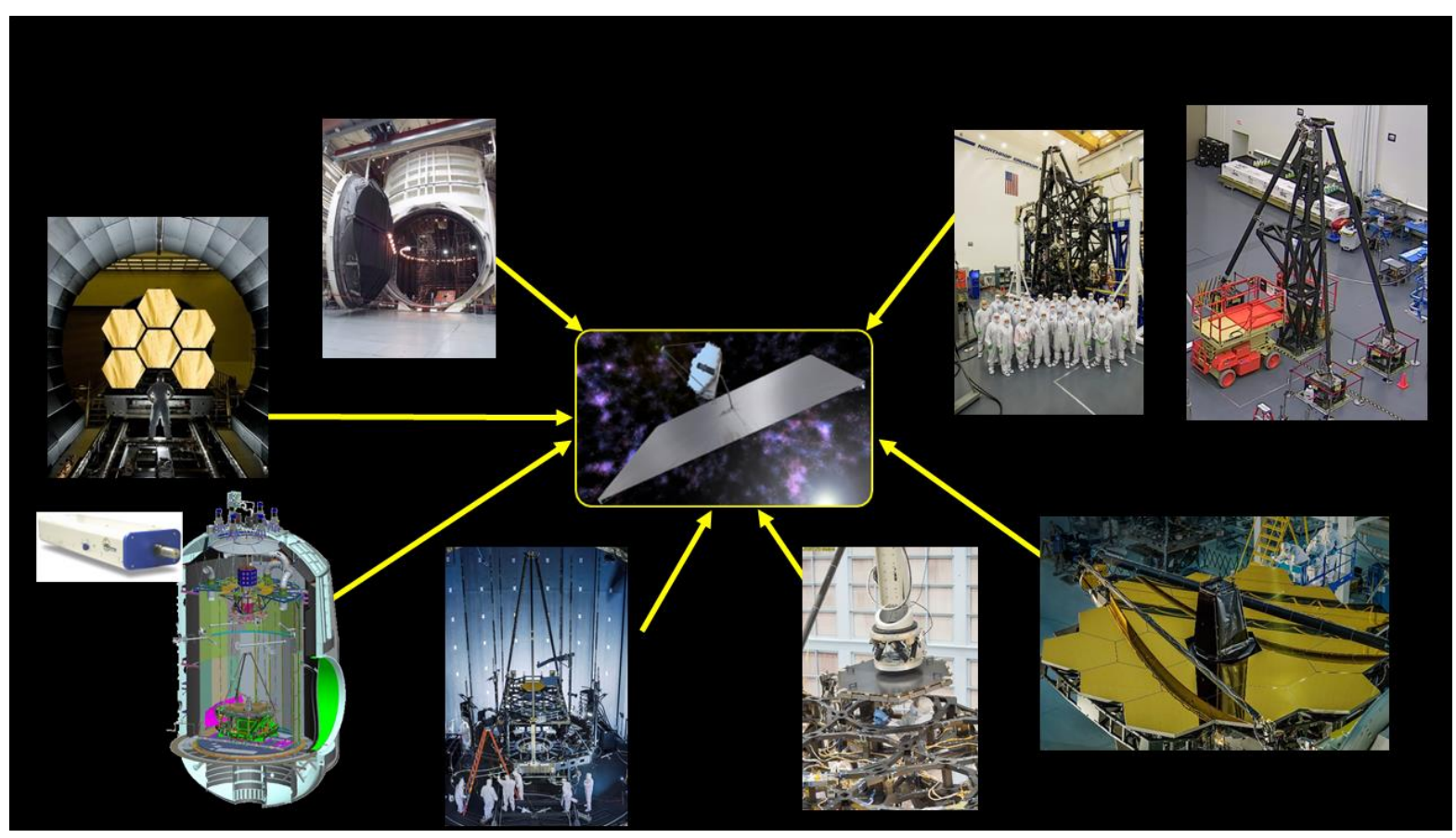

Figure 12: JWST Heritage That Can Reduce Risk and Cost 


\section{CONCLUSION}

Based on several years of ATLAST studies, we have attempted to provide a complete end to end look at what a large Ultra-Violet Optical Infrared Observatory would look like. While other observatory architectures are feasible, we believe the approach outlined balances the need for general astrophysics and Exoplanet detection. While we anticipate future changes and evolution, starting to integrated the telescope with the coronagraph and instruments is an important step that can feed into yield calculations and future trade studies.

\section{REFERENCES}

\footnotetext{
${ }^{i}$ Feinberg, L. D., Jones, A., Mosier, G., et al. 2014, in Society of Photo-Optical Instrumentation Engineers (SPIE) Conference Series, Vol. 9143, 16

ii Redding, D. C., Feinberg, L., Postman, M., et al. 2014, in SPIE, Vol. 9143,12

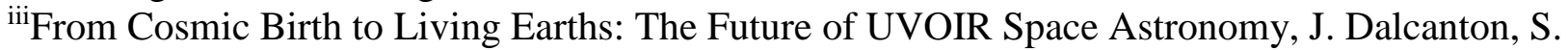
Seager et al, arXiv:1507.04779

iv Stark, C. C., Roberge, A., Mandell, A., et al. 2015, ApJ, 808, 149

${ }^{v}$ Feinberg, L. D, et al, Modular Assembled Space Telescope, 2015, Optical Engineering, 52 (9)

${ }^{v i}$ Stahl, H. P., et al, 2004, in SPIE, Vol 5487 (31)

vii https://www.zygo.com/met/absolutepositionmeasurement/ZPS_Specs_Sheet.pdf

viii N'diaye, $\mathrm{M}$ et al, Apodized Pupil Lyot Coronagraphs for Arbitrary Apertures V. Hybrid Shaped Pupil Designs for Imaging Earth-like planets with Future Space Observatories, arXiv:1601.02614

viii Eisenhower, M ATLAST ULE mirror segment performance analytical predictions based on thermally induced distortions

viii Babak Saif et al, "New method for characterizing the state of optical and opto-mechanical systems ", Proc. SPIE 9143, Space Telescopes and Instrumentation 2014: Optical, Infrared, and Millimeter Wave, 91430C (August 2, 2014);
} 